\title{
Optimal Survey Design for Offshore Deformation Monitoring
}

\author{
Martin W. Rayson ${ }^{1, *}$, Noor Safaruddin Kamaruddin ${ }^{2, \dagger}$, and Mohd Razali Mahmud ${ }^{2, \ddagger}$ \\ ${ }^{1}$ WX Geo Services LLC, Houston, Texas, USA. \\ ${ }^{2}$ Faculty of Geoformation \& Real Estate (FGHT), UTM, Jalan Ilmu, 81310 Skudai, Johor Bahru, Johor, Malaysia.
}

\begin{abstract}
Monitoring deformation experienced by facilities engaged in offshore hydrocarbon extraction is important to understanding any structural integrity changes a production and development asset may suffer. Facilities can be platform or seabed based depending upon water depth and environment in which the assets are situated. There have been some well document case studies showing different survey techniques being used to undertake such monitoring campaigns in a variety of different settings, and reference is made to these publications.

This research takes a step back to examine the problem from a Geomatics perspective. This is achieved by firstly, developing mathematical model(s) showing all the deformation parameters a facility will experience and secondly, determining an optimal survey design that will measure the deformation parameters to required quality measure tolerance. This includes consideration of coordinate system usage and the actual survey observation campaign. The optimal observation scheme is described by the selection of where survey control stations will be located, and the observations made between the stations to measure the deformation parameters of the model. Next, consideration is given to the error budget the observations must operate within to develop the stochastic model incorporate into the survey design. The dynamic deformation model must include the rates of change a facility is expected to experience within each of the directional vectors of the model (e.g. pitch, roll, yaw and heave) and the statistical tolerance to which they are required.

The results of the survey design will compute aposteriori matrices from which the classical quality measures are derived. The computational process enables multiple scenarios to be examined to help determine optimal observation schemes for individual facilities in different offshore environments. Finally, current technologies are examined to determine which are best suited to measure the rates of change built into the deformation model to the quality tolerances specified. The final observation scheme will recommend an optimal combination of technologies to best determine subsidence rates for different offshore environments.
\end{abstract}

\section{Introduction}

Subsidence and deformation, associated with offshore installations, results from the compaction of overburden stratigraphy, because of the extraction of hydrocarbons from the reservoirs of the field. The amount of subsidence experienced is linked to several critical factors, including type of reservoir rock and depth of the reservoir below seabed. Typically, the design of any offshore platform, or facility, will factor in an expected level of subsidence to ensure a safe air gap between production decks and mean sea level. Monitoring subsidence rates is critical to ensure that the rates experienced do not exceed the rates factored into the design.

Typically, subsidence has been monitored using single technologies, e.g. GNSS, acoustic networks, pressure sensors etc. To optimally monitor the dynamics experienced, one needs to look at a more holistic approach, that factors in deformation in addition to pure vertical subsidence. This results in a multi-dimensional model that estimates rates of change of the various components of the model with respect of time.

The main scope of the research has been the development of a generic mathematical model that describe the various deformation components, the expected rates of change each component might experience and the quality

\footnotetext{
${ }^{*}$ Corresponding author: martin.rayson@geomaticsolutions.com

+ Email: safaruddin@graduate.utm.my

‡ Email: razalimahmud@utm.my
} 
to which the parameters are required (due to hydrocarbon extraction). Having determined the generic models, it is possible to determine what optimal survey design should be deployed to measure the deformation being experienced.

This includes to location of survey control points on the facility, plus the observation scheme to measure deformation. The design must also factor in the stochastic model associated with the various observation types incorporated into the observation scheme. This ensures that there is a thorough understanding that the proposed observations can make to the accuracy / precision such that it is on par with the rates of change included within the generic models.

Using a classical best linear unbiased estimator, an adjustment technique (LSA) is performed using the design matrix (functional model) and stochastic model proposed as part of the survey design (Cross 1983). Of specific interest are the quality measures extracted from the aposteriori covariance matrices computed by the computations. This enables both precision and reliability measures to be determined in the form of horizontal error ellipses and marginal detectable errors respectively. Both are used to gauge the usefulness of the proposed survey design at achieving the objectives of the observation campaign.

Survey design, using these types of approaches is nothing new (Rayson 1990). Using such computations to conduct these types of investigations is well document in the literature reviews performed, for a host of different survey campaigns, whether it be measuring deformation of civil engineering structures (dams/bridges) or plate tectonic motion and earthquakes. However, its application within deformation monitoring of offshore facilities is a novel approach. To date, when subsidence monitoring has been conducted it is done so with a specific bias towards certain equipment types, e.g. GNSS only. However, using just one type of survey observation is unlikely to yield an optimal solution. Optimization comes from the careful consideration of combination technologies to observe the rates of change along specific vectors. Using the design models indicated, it will be demonstrated that optimal survey design schemes can be derived for different offshore environments.

\section{Methodology}

An offshore facility, associated with hydrocarbon extraction and recovery, has a high likelihood of experiencing some level of deformation and subsidence during the life of the field. As hydrocarbons are extracted from the reservoir the porous spaces between the rock grains become depleted of fluids to a certain degree. Weight of the overburden stratigraphy, will cause a compaction of the reservoir lithology, which in turn will account for subsiding of the seabed above the main structure of the reservoir and thus the associated facility will experience deformation. The type of offshore facility installed will depend upon environment factors such as water depth and subsea engineering requirements. The amount of deformation the facility will endure will depend upon the type of reservoir rock and depth of reservoir below seabed. Other geological and environmental factors must also be considered to develop an overall model of the forces that impact the offshore facility (Dudley, van der Linden, Mah 2009).

The optimal measurement of deformation must consider several environmental and engineering factors associated with the facility of interest. A computer design process is considered that follows a basic four stage design process (Rayson and Cross 1990) that considers the following criteria:

- Development of a basic deformation model a facility may experience that considers the parameters associated with the deformation.

- Specification of the precision and reliability quality measures to which the deformation parameters are required.

- Design of the optimal observation scheme that meets the tolerances required for an individual facility.

Design schemes for deformation monitoring have been examined over the last few decades and are well documented (Hatchell et. al. 2017, Miandro et. al. 2017, Sahabi et. al. 2006, Vilaseca et. al. 2016)

\subsection{General parameters of deformation model}

The general parameters of the deformation model a facility will experience are modelled in terms of three translation parameters and three rotational parameters, all with respect to time, to show motions in terms of heave, yaw, pitch and roll. The example given examines the forces experienced on a platform sitting on a jacket. 


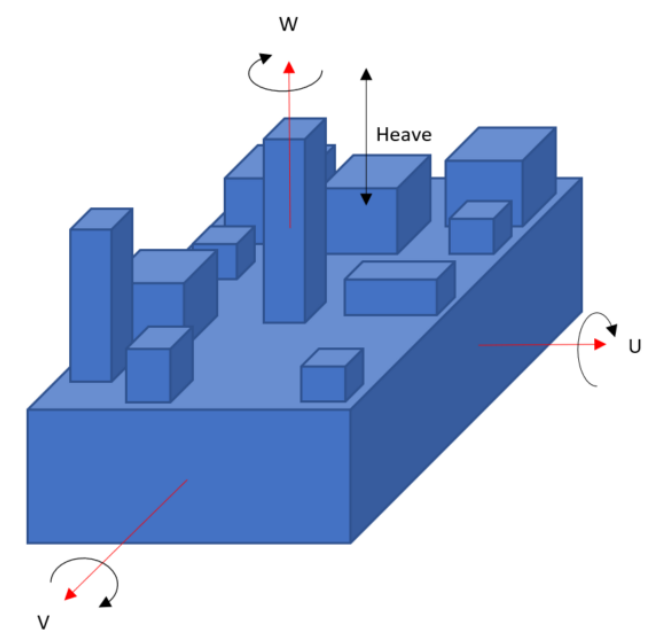

Fig 1. Four major motions effecting a facility

The model developed incorporate both rotational and linear parameters represented in the matrix expression shown in equation (1). It includes all the translation and rotation parameters with respect to time. The model is increased in complexity by introducing other forces, such as stress. However, it is basic form the model will take on the following generic form:

$$
\begin{array}{r}
f\left(\Delta U, \Delta V, \Delta W, \theta_{u}, \theta_{v}, \theta_{w}\right)=0 \ldots \ldots(1) \\
{\left[\begin{array}{c}
U^{\prime} \\
V^{\prime} \\
W^{\prime}
\end{array}\right]_{t_{1}}=H+\left[\begin{array}{c}
\Delta U \\
\Delta V \\
\Delta W
\end{array}\right]+\left[\begin{array}{ccc}
1 & -\theta_{W} & \theta_{U} \\
\theta_{W} & 1 & -\theta_{V} \\
-\theta_{U} & \theta_{V} & 1
\end{array}\right]\left[\begin{array}{c}
U \\
V \\
W
\end{array}\right]_{t_{0}}}
\end{array}
$$

Where:

- Rotational parameters are indicated by: $\theta_{V}, \theta_{U}, \theta_{W}$.

- Translation parameters are indicated by: $\Delta U, \Delta V, \Delta W$

- Heave parameter represents any subsidence and is represented by $\mathrm{H}$

- A series of control stations will comprise the survey network. The coordinates of the control stations at different epochs are described by $U, V, W$ and $U^{\prime}, V^{\prime}, W^{\prime}$.

- Time is represented in epochs to enable rates of change to be established.

For later use, the matrix expression is expanded to derive the following three expressions:

$$
\begin{gathered}
U_{t_{1}}^{\prime}=H+\Delta U+\left(U_{t_{0}}-V_{t_{0}} \theta_{W}+W_{t_{0}} \theta_{U}\right) \\
V_{t_{1}}^{\prime}=H+\Delta V+\left(U_{t_{0}} \theta_{W}+V_{t_{0}}-W_{t_{0}} \theta_{V}\right) \\
W_{t_{0}}^{\prime}=H+\Delta W+\left(-U_{t_{0}} \theta_{U}+V_{t_{0}} \theta_{V}+W_{t_{0}}\right)
\end{gathered}
$$

Where, generally, the deformation parameters $(\Phi)$ to be estimated from the survey design are described by:

$$
\Delta \hat{x}=\Phi(\phi)
$$

Where $\phi$, is the vector of deformation parameters.

To measure the general deformation parameters described in equations (2-6) requires an appropriate observation scheme be designed comprising a series of survey measurements are made between a strategically located set of control stations. The selection of stations and observations constitute the survey design. The number of observations and duration over which they are made will constitute the survey campaign. Considerations must also be given to whether the survey campaign will be discrete or continuous, or a mixture of both. The observations 
will enable changes in the control station coordinates to be determined, from which the rotations, translations and subsidence can be obtained.

\subsection{Coordinate systems}

Consideration must be given to the coordinate system within which the survey design and survey campaign are conducted. Preference is given to operating within a local topocentric system, which is intrinsically tied to the offshore facility whose deformation is being monitored. Furthermore, a geographic 3D coordinate reference system is selected whose relation and transformation to the local topocentric coordinate system is known and regulated. The geographic 3D CRS selected in all scenarios conducted is WGS 84.

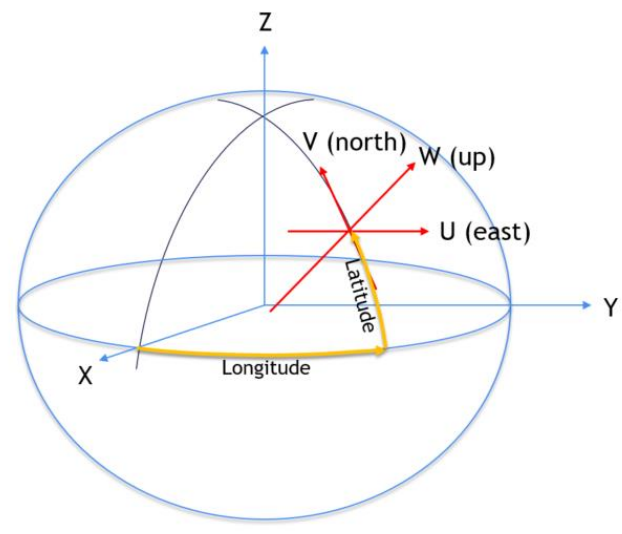

Fig. 2. Local topocentric to Geographic coordinate systems

Converting between geographic coordinate system and local topocentric (LT) system is performed using the following equations (6-8) as recommended by IOGP guidance note 373-07-02 (IOGP 2017):

$$
\begin{gathered}
U=(v+h) \cos \varphi \sin \left(\lambda-\lambda_{0}\right) \\
V=(v+h)\left[\sin \varphi \cos \varphi_{0}-\cos \varphi \sin \varphi_{0} \cos \left(\lambda-\lambda_{0}\right)\right]+e^{2}\left(v_{0} \sin \varphi_{0}-v \sin \varphi\right) \cos \varphi_{0} \\
W=(v+h)\left[\sin \varphi \sin \varphi_{0}+\cos \varphi \cos \varphi_{0} \cos \left(\lambda-\lambda_{0}\right)\right]+e^{2}\left(v_{0} \sin \varphi_{0}-v \sin \varphi\right) \sin \varphi_{0}-\left(v_{0}+h_{0}\right)
\end{gathered}
$$

All symbols have their usual meaning, where:

$\varphi_{o}, \lambda_{0}, h_{0}$ are geographic coordinates for the origin of the LT coordinate system.

$v$ is the radius of curvature in the prime meridian at the latitude of the point being converted.

$v_{o}$ is the radius of curvature in the prime meridian at the latitude of the origin of the LT system.

$$
\varphi=\frac{a}{\left(1-e^{2} \sin ^{2} \varphi\right)^{1 / 2}}
$$

Where:

$a$ is the semi major axis of the WGS 84 reference ellipsoid.

$e^{2}$ is the eccentricity (squared) of the WGS 84 reference ellipsoid.

\subsection{Survey network design}

Survey network design concerns itself with the construction of an optimal combination of control stations and observations, over the facility, that will adequately measure the rates of change of the deformation parameters of the general model (Cross 1981, Rayson 1990). As all facilities have different characteristics a survey network design is required for each. The design must take into consideration limiting factors about each facility that will 
limit where control stations can and cannot be located and between which control stations observations can be made.

Determining where to locate survey control stations must consider factors that include:

- Types of survey observations being made. For example, is line of sight required, good sky visibility, no electrical interference, stability to give sustainability, etc.

- Restrictions the facility may impose upon where stations can be located (zones of operation), for example, restrictions on where certain equipment types can or cannot be operated.

To assist with the survey design, a 3D model of the facility is utilized in a computer aided design (CAD) software application. Various design permutations can thus be examined to optimize the design of the survey campaign, working within the logistical limitations imposed.

The survey network design determines between which control stations observations are made to measure the general dynamic variables. Within the CAD application the proposed observation scheme is described by relating to the unknown parameters to the observable using the classical observation equations, of which the ones for range, bearing and height are shown in equations $10-12$ :

$$
\begin{gathered}
r=\left[\left(U_{2}-U_{1}\right)^{2}+\left(V_{2}-V_{1}\right)^{2}+\left(W_{2}-W_{1}\right)^{2}\right]^{1 / 2} \\
\theta=\tan ^{-1} \frac{\left(U_{2}-U_{1}\right)}{\left(V_{2}-V_{1}\right)} \\
\Delta W=\sin \theta
\end{gathered}
$$

The observations that comprise the survey campaign are collated into the design matrix, known as the functional model (A). Within the matrix, there is one row for each observation in the network and one column for each unknown parameter (Cross 1983, Bagherbandi 2016). The elements of the design matrix are a series of partial differentials, derived from the observation equations, that describe the relationship between the observation being made and the unknown parameter to be determined.

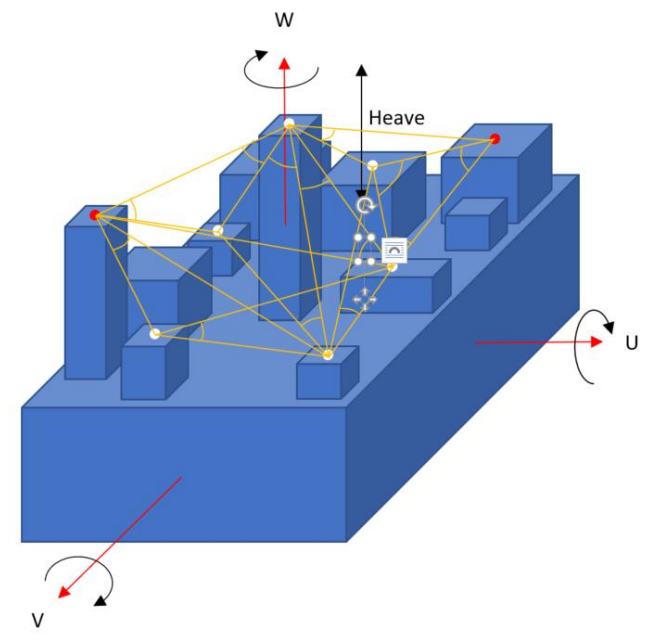

Fig. 3. Survey network design

The network requires scale and orientation to have any physical meaning. This is achieved using either:

- Two known control stations whose coordinates are determined from survey observations taken external to the facility, e.g. GNSS precise point positions or GNSS vectors.

- One known control station (determined in same manner as above) and one survey bearing, e.g. some form of gyro compass observation.

The functional model is described in the following generic fashion: 


$$
A=\left[\begin{array}{ccc}
\frac{\partial F_{1}}{\partial x_{1}} & \cdots & \frac{\partial F_{1}}{\partial x_{n}} \\
\vdots & \ddots & \vdots \\
\frac{\partial F_{n}}{\partial x_{1}} & \cdots & \frac{\partial F_{n}}{\partial x_{n}}
\end{array}\right]
$$

For example, for a range observation, made between two control stations, the following partial derivates are used to describe their relationship.

$$
r_{1}=\left[\left(U_{1}-U_{2}\right)^{2}+\left(V_{1}-V_{2}\right)^{2}+\left(W_{1}-W_{2}\right)^{2}\right]^{1 / 2}
$$

Therefore:

$$
\frac{\delta r_{1}}{\delta U_{1}}=\frac{\left(U_{1}-U_{2}\right)}{r_{1}}
$$

The same can be generated for the relationships between $r_{1}$ and the remaining five unknown parameters, $V_{1}, W_{1}, U_{2}, V_{2}, W_{2}$. This is a three-dimensional model considering both horizontal and vertical components of the general model.

All observations made in a survey campaign contain error, which as a minimum will be random error. Such errors are associated with components of the observation such as atmospheric error and instrument error, all of which are not measurable, but can have their sizes estimated with a certain degree of probability. This is described using the standard deviation term $\left(\sigma_{x}\right)$, with the probability typically set to one sigma, or $68 \%$ probability of the error being of that size or smaller. When an observation scheme is designed such errors are formally described within an apriori matrix known as the covariance matrix of the observations. The components of such a matrix are described in the following way:

$$
\left.C_{l}=\left[\begin{array}{ccc}
\sigma_{1}^{2} & \cdots & \sigma_{1 n} \\
\vdots & \ddots & \vdots \\
\sigma_{n 1} & \cdots & \sigma_{n}^{2}
\end{array}\right]\right]
$$

The diagonal components of the matrix describe the variance terms, which are the standard errors multiplied by itself for each observation. The off-diagonals describe the covariance terms, which describe the errors shared between pairs of observations because of perhaps them being made at the same time. Although in principle, these terms could be derived, in theory they are often ignored and are set to zero due to the complexity of their modelling. From the covariance matrix of the observations, the stochastic model (W) is computed as follows:

$$
W=\frac{1}{C_{l}}=C_{l}^{-1}
$$

The survey design does not use any actual observations, but only a proposed scheme that addresses the specific survey problem. Modifications to the scheme will enable an optimal design to be determined that will best answer the problem. In this instant, what combination of survey stations and survey observations will best enable the unknown variables for the facilities dynamics to be measured, e.g. rotations and translations, to the rates to change predicted. To determine the optimal combination a computation must be conducted whose answers, when interpreted, will lead to a suitable campaign being designed. This computational process is not a full blown LSA solution but a reduced version of it. The computation will enable a series of precision and reliability measures to be derived from the following matrix computations:

$$
\begin{gathered}
C_{\hat{x}}=\left[A^{T} W A\right]^{-1} \\
C_{\hat{v}}=W-A\left[A^{T} W A\right]^{-1} A^{T}
\end{gathered}
$$

- $C_{\hat{x}}$ is known as the covariance matrix of the unknown parameters.

- $C_{\hat{v}}$ is known as the covariance matrix of the residuals.

- All other symbols have their usual meanings.

For example, equation (18) is used to derive a horizontal error ellipse for each unknown station contained within the survey design scheme. It is accepted that all observations contain error, therefore it is accepted that all unknown station coordinates, derived from the observations, will also contain error. This is known as gaussian law of propagation (Cross 1983). 


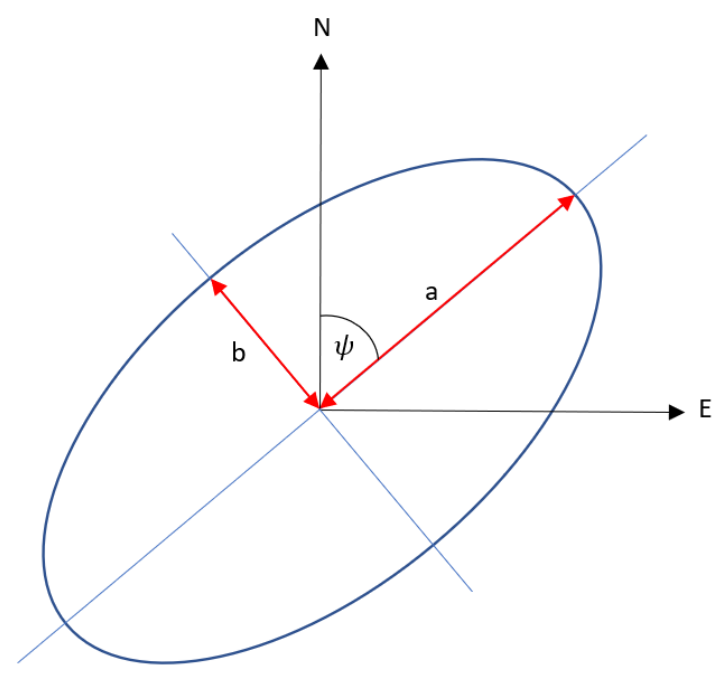

Fig. 4. Horizontal error ellipse

Put in its basic sense, the horizontal error ellipse is a quality measure used to describe the probability of a computed horizontal position falling within the foot print of the ellipse boundary, with a specified probability (typically, 2 sigma $(2 \sigma), 95 \%)$.

The two key parameters associated with this quality figure are semi-major axis and the orientation. This is largely dictated by the geometry of the observation scheme.

A marginal detectable error (MDE), describes the chances of detecting an observational error to a certain probability. It is controlled by the power of the test, through hypothesis theory. Furthermore, it is important to know what effect an undetected error will have upon the unknown deformation parameters. This is known as the external reliability test. Both constitute reliability measures and are largely driven by the redundancy of the observation scheme. Redundancy is described by the difference between the number of observations made and the number of unknown parameters to be solved (Cross 1983).

Optimization requires that both precision and reliability measures are reduced to a required tolerance level. This is a critical part of a campaign design and is crucial to the success of monitoring a facilities deformation. Without proper consideration a campaign will not succeed in its objectives.

\subsection{Covariance matrix of deformation parameters}

From equation (6) an expansion can be taken to show the relationship between the deformation parameters required and covariance matrix of the unknown parameters computed from equation (20). By forming this expansion, the precisions of the deformation parameters can be expressed by:

$$
C_{\widehat{\phi}}=\left(f^{T} Q f\right)^{-1}
$$

Where:

$f=$ functional model of deformation parameters, derived from:

$$
f=\left[\frac{d \phi}{d \Delta x}\right] \Delta x=\Delta \hat{x}
$$

$Q=$ stochastic model derived from:

$$
C \Delta \hat{x}^{-1}
$$

As the stochastic model is the inverse of the covariance matrix from equation (22), the stochastic model of the coordinate differences is given by: 


$$
Q=C_{\hat{x}_{2-1}}^{-1}
$$

Hence:

$$
Q=C_{\hat{x}}^{-1}
$$

Therefore:

$$
Q=\frac{1}{2} A^{T} W A
$$

\subsection{Monitoring deformation}

Once the generic deformation model has been linked to the optimal survey design, the next stage is to determine which technologies are best suited to measure the rates of change of the deformation parameters to the quality measure tolerances. Environmental factors play a crucial understanding in which observations are best suited to solve the problem. All technologies have their limitations, but a controlled combination will yield the best solution for a specific solution. Therefore, taking into consideration environmental matter will greatly assist the design processes.

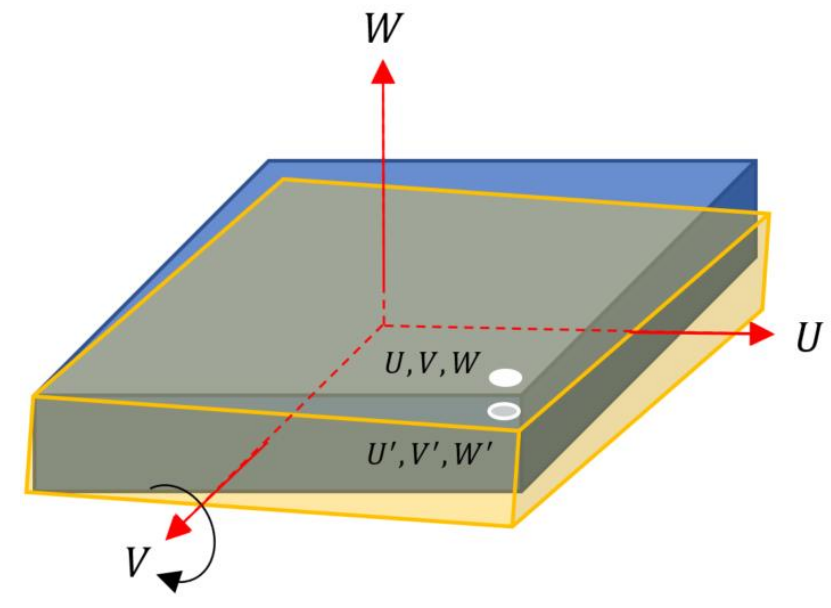

Fig. 5. Deriving roll rate, an example

The initial observation campaign enables the 'baseline' survey to be conducted. This will determine the coordinates of each control station in the local topocentric coordinate system. This is considered time zero. Subsequent campaigns, conducted at time $t_{x}$ will determine a new set of control station coordinates. Differences between the two sets of coordinates will enable motions to be determined. Figure 5 illustrates a simple example of a facility experiencing roll motion. There is a change in control station coordinates between the various observation epochs which is described in equation (20).

$$
x_{t_{2-1}}=\left[\begin{array}{ll}
I & -I
\end{array}\right]\left[\begin{array}{l}
x_{2} \\
x_{1}
\end{array}\right]
$$

Looking at this from the perspective of the covariance matrices:

$$
C \Delta \hat{x}_{t_{2-1}}=\left[\begin{array}{ll}
I & -I
\end{array}\right]\left[\begin{array}{cc}
C_{\hat{x}_{2}} & 0 \\
0 & C_{\hat{x}_{1}}
\end{array}\right]\left[\begin{array}{c}
I \\
-I
\end{array}\right]
$$

When multiplied out the expression becomes:

$$
C \Delta \hat{x}_{t_{2-1}}=C_{\hat{x}_{2}}-C_{\hat{x}_{1}}
$$

If the observation campaign is the same between both epochs equation (29) reduces to:

$$
C \Delta \hat{x}_{t_{2-1}}=2 C_{\hat{x}_{1}}
$$


Therefore, quality measures can be associated with the deformation parameters using the approaches described.

\section{Result and Discussion}

This study demonstrates, from a geomatics perspective, a methodology to determine what combination of survey control stations and observations, through an optimal survey design, are best suited to measure deformation parameters within specific offshore environments. Next, it demonstrates that a rigorous computation analysis tool is available to derive the quality measures associated with the deformation parameters. This enables unique survey designs to be computed for any offshore facility.

A robust survey design thus offers the most ability to choose the most suitable combination of technologies for making the observations included within the survey design to derive the rates of changes of the deformation parameters. This includes all current off-the-shelf technologies such as, GNSS, acoustic networks, InSAR, airgap and traditional precise levelling (Fujita et. al. 2006, Davis et. al. 2000). Consideration is also given to logistical and cost related matters. Certain technologies have limitations in their use, implementation and deployment within the environments proposed.

\section{Concluding Remarks}

Through careful consideration of the four stages of the network design process an optimal observation campaign can be reported at designated epoch intervals to measure the rates of change associated with the deformation parameters contained within the general model. As all offshore facilities differ in design the tool will offer the flexibility to create unique designs to suite each facility. Such flexibility removes the likelihood of generic solutions being used that only utilize single solution technologies.

\section{References}

1. Bagherbandi, M., Deformation monitoring using different least squares adjustment methods: A simulated study. KSCE Journal of Civil Engineering, 20(2), pp.855-862, (2016)

2. Cross, P.A, 1983. Advanced least squares applied to position-fixing. North East London Polytechnic, Department of Land Surveying. Working paper no.6: 205pp.

3. Cross, P.A., 1981. Computer aided design of geodetic networks. Proceedings of VI International Symposium on Geodetic Computations, Munich 12pp.

4. Davis, E., Wright, C., Demetrius, S., Choi, J. and Craley, G., Precise tiltmeter subsidence monitoring enhances reservoir management. In SPE/AAPG Western Regional Meeting. Society of Petroleum Engineers., January 2000.

5. Dudley, J.W., van der Linden, A. and Mah, K.G. Predicting accelerating subsidence above the highly compacted Luconia carbonate reservoirs, offshore Sarawak Malaysia. SPE Reservoir Evaluation and Engineering., 12(01), pp 104-115, February 2009.

6. Fujita, M., Ishikawa, T., Mochizuki, M., Sato, M., Toyama, S.I., Katayama, M., Kawai, K., Matsumoto, Y., Yabuki, T., Asada, A. and Colombo, O.L., GPS/Acoustic seafloor geodetic observation: method of data analysis and its application. Earth, planets and space, 58(3), pp.265-275, 2006.

7. Hatchell, P., de Vries, R., Gee, V., Cousson, H., Lopez, J., Dunn, S., Street, N., Parsons, A., Cheramie, J. and Fischer, E., October. Seafloor deformation monitoring: Past, present, and future. In 2017 SEG International Exposition and Annual Meeting. Society of Exploration Geophysicists. 2017, pp. 5233-5238.

8. Kutoglu, H.S. and Berber, M., Optimal number of reference points in deformation monitoring. Acta Geodaetica et Geophysica, 50(4), 2015, pp.437-447.

9. Miandro, R., Dacome, C., Mosconi, A. and Roncari, G., An Innovative Approach for Offshore Subsidence Monitoring: Technology Scouting, Feasibility Studies and Realization. In Offshore Mediterranean Conference and Exhibition. Offshore Mediterranean Conference, May 2017.

10. Naankeu-Wati, G., Geldof, J.B. and Seube, N., Error Budget Analysis for surface and underwater survey system. The International Hydrographic Review, (15)., 2017, pp. 21-46.

11. Sahabi, H., M. A. Rajabi, and J. A. R. Blais. Optimal Design of Deformation Monitoring Networks Using Genetic Algorithm. Oral presentation at the Fifth International Symposium Turkish-German Joint Geodetic Days in Berlin, Germany. 2006. 
12. Rayson, M.W. Optimal design of survey networks for monitoring earthquake prediction. Ph.D. Thesis, University of Newcastle. 1990.

13. Rayson, M.W. and Cross, P.A. Computer Aided Design of optimal networks for monitoring crustal tectonic activity. P. Vyskocil et al. (eds.), Global and Regional Geodynamics. Springer-Verlag New York Inc. 1990, pp 262-275.

14. Report 373-07-2. Coordinate conversions and transformations including formulas. IOGP Guidance Note. 2017.

15. Vilaseca, G., Deplus, C., Escartín, J., Ballu, V., Nomikou, P., Mével, C. and Andreani, M., Oceanographic Signatures and Pressure Monitoring of Seafloor Vertical Deformation in Near-coastal, Shallow Water Areas: A Case Study from Santorini Caldera. Marine Geodesy, 39(6), 2016, pp.401-421. 